\title{
Thrombotic thrombocytopenic purpura: reducing the risk?
}

\author{
Michael C. Berndt ${ }^{1}$ and Robert K. Andrews ${ }^{2}$
}

${ }^{1}$ Biomedical Diagnostics Institute, Dublin City University, and Royal College of Surgeons in Ireland, Dublin, Ireland. ${ }^{2}$ Australian Centre for Blood Diseases, Department of Clinical Haematology, Monash University, Alfred Medical Research and Education Precinct (AMREP), Melbourne, Victoria, Australia.

\begin{abstract}
von Willebrand factor (vWF) has a key role in initiating platelet aggregation, and thereby thrombus formation, that is dependent on its ability to form appropriately sized multimers. Ultralarge multimers promote the formation of the microvascular thrombi that are hallmarks of the life-threatening condition thrombotic thrombocytopenic purpura (TTP). In this issue of the JCI, Chen et al. show that the drug $\mathrm{N}$-acetylcysteine (NAC) can decrease the size of $\mathrm{VWF}$ multimers in vitro and in vivo, resolving thrombi in mice. These data suggest that NAC could potentially be used to treat thrombotic conditions such as TTP.
\end{abstract}

vWF is a large protein that functions as a multimer in the complex process of hemostasis. Some thrombotic diseases are associated with the formation of ultra-large $\mathrm{vWF}$ multimers (ULVWF). Notable among such diseases is thrombotic thrombocytopenic purpura (TTP), where ULVWF result from acquired or congenital abnormalities of the physiological regulator of vWF multimer size, a disintegrin-like and metalloproteinase with thrombospondin repeats-13 (ADAMTS13). The life-threatening condition is characterized by acute episodes of microangiopathic hemolytic anemia and thrombocytopenia, with or without renal failure or neurologic abnormalities, and an approximate mortality rate of $20 \%$ (1). Current frontline treatment involves protracted plasma exchange, with its associated complications, and in some patients, adjuvant treatment with corticosteroids (1). There is thus a clear clinical need for better therapeutic approaches for the treatment of TTP. In this issue of the JCI, Chen and colleagues (2) report that $\mathrm{N}$-acetylcysteine (NAC), a free thiol-containing compound used for the treatment of chronic obstructive lung disease and as an antidote for toxicity due to acetaminophen, reduces intra- and intermolecular disulfide bonds in both human and mouse vWF, decreasing its multimer size and downregulating vWF function (Figure 1). Importantly, the use of NAC in patients with either chronic

Conflict of interest: The authors have declared that no conflict of interest exists.

Citation for this article: J Clin Invest. 2011; 121(2):522-524. doi:10.1172/JCI46091. obstructive lung disease or acetaminopheninduced toxicity is not associated with bleeding, suggesting that NAC (or related compounds) could potentially be used in the treatment of thrombotic diseases associated with ULVWF such as TTP.

\section{vWF and thrombosis}

vWF is a key adhesive protein in the vasculature that enables circulating, resting platelets in flowing blood to become localized and initiate platelet aggregate (thrombus) formation $(3,4)$. Multivalent vWF multimers support the initial adhesion of circulating platelets to an injured blood vessel where subendothelial matrix is exposed or a diseased vessel where endothelial cells are activated. This involves an interaction between vWF and the primary platelet receptor, glycoprotein Ib $\alpha$ (GPIb $\alpha$ ), the ligand-binding subunit of the GPIb-IX-V complex. Engagement of GPIb-IX-V leads to activation of the platelet integrin $\alpha_{\text {IIb }} \beta_{3}$ (GPIIb-IIIa), which binds to vWF or other ligands, such as fibrinogen, and mediates platelet aggregation. The interaction between GPIb $\alpha$ and vWF is adapted to function at elevated shear stress, as occurs at high arterial shear rates or at pathological shear within a stenosed artery, with the binding domains on both the receptor and ligand undergoing conformational activation to enhance adhesion $(5,6)$.

Synthesis and secretion of vWF in megakaryocytes/platelets or endothelial cells starts from a complex gene of $178 \mathrm{~kb}$ and 51 introns. The gene encodes a precursor protein that initially assembles as a large disulfide-linked, tail-to-tail, dimeric progenitor, followed by cleavage of the pro-domain, and formation of head-to-head disulfide-linked multimers of $20,000 \mathrm{kDa}$ or more (4). Each subunit of approximately $275 \mathrm{kDa}$ comprises domains D'-D3-A1-A2-A3-D4-B1-B2B3-C1-C2. The A1 domain (vWF-A1) contains the GPIb $\alpha$-binding site, the A2 domain contains the ADAMTS13 cleavage site, the A3 domain contains a collagen-binding site, and the $\mathrm{C} 1$ domain contains an Arg-Gly-Asp (RGD) sequence that binds $\alpha_{\mathrm{IIb}} \beta_{3}$.

\section{vWF-A1 binds GPIb $\alpha$}

The vWF-A1 domain was identified as the GPIb $\alpha$-binding site using biochemical approaches more than 20 years ago. Proteolytic digestion of purified human vWF by trypsin or dispase generated a disulfide-linked homodimeric 52/48-kDa fragment (7) or a 39/34-kDa monomeric fragment (8), respectively, that bound to GPIb $\alpha$ and blocked binding of vWF (fragments had two molecular weights due to variable glycosylation). These analyses provided evidence that (a) intermolecular disulfide bonds involved in forming vWF multimers were present within the 52/48$\mathrm{kDa}$ fragment (based on the dimeric tryptic fragment) and (b) that vWF-A1 contained an intramolecular disulfide bond (based on the apparent molecular weight of the 39/34-kDa fragment under nonreducing and reducing conditions). Amino acid sequencing showed that this intramolecular disulfide bond must involve Cys residues C1272-C1458 (C509-C695 in the mature sequence). Subsequent analysis of crystal structures of GPIb $\alpha / v W F-A 1$ interactive domain complexes, mutagenesis studies examining this interaction under shear conditions, and in silico modeling of individual receptor/ligand bonds have provided detailed understanding of how vWF can support platelet adhesion under shear conditions $(5,6,9-11)$. This involves conformational activation of shear-dependent catch-slip bonding. In catch bonds, force enhances the binding interaction to prolong bond lifetime due to conformational 

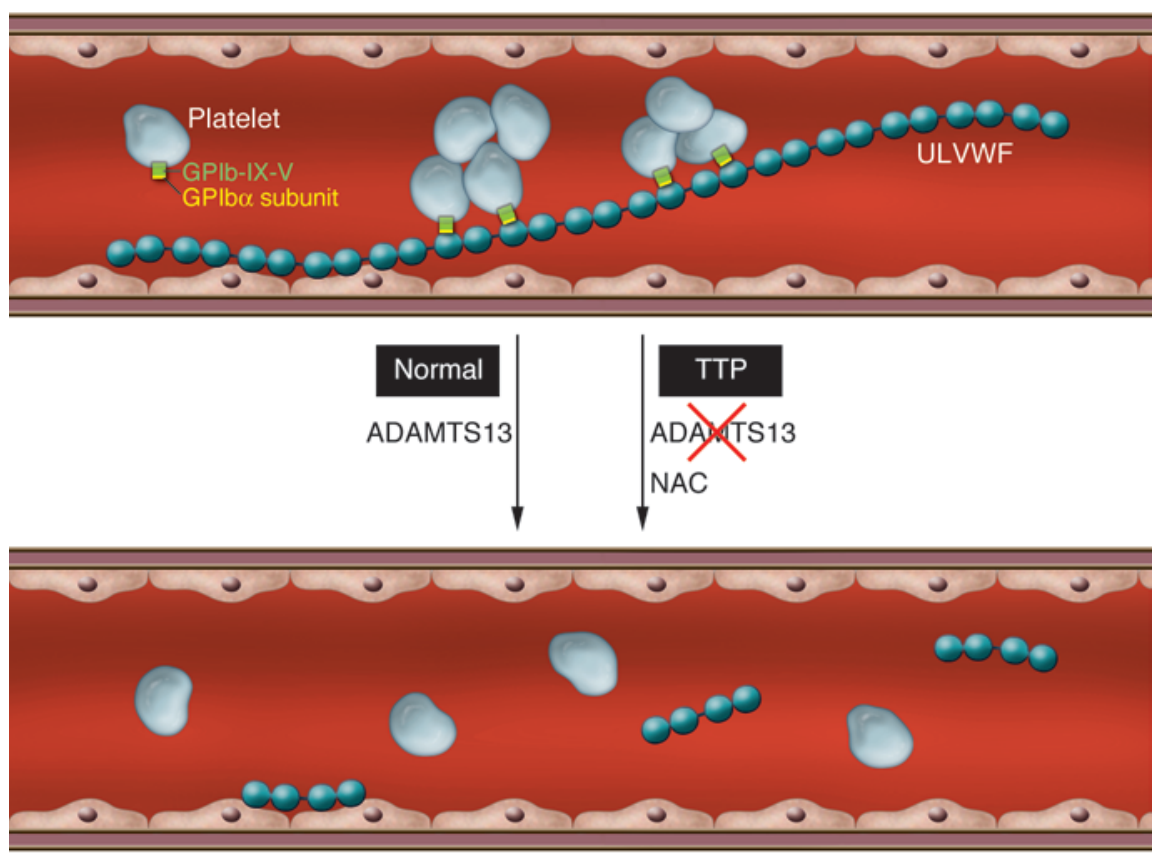

changes; in slip bonds, force lowers the energy barrier to shorten bond lifetime. The catch/slip bonds and strength of the interaction thus regulate attachment/detachment, enabling the circulating platelets to roll, firmly adhere, or detach depending on shear force $(5,6)$. ULVWF multimers expressed on activated endothelial cells are highly prothrombotic and also bind platelets with high avidity (12). What is also now clear is that the mechanisms by which vWF multimer composition is regulated are critical for normal or pathological vWF function $(3,4,13)$ and that therapeutic control of aberrant vWF multimerization could have a major impact on clinical conditions such as TTP.

\section{ADAMTS13 and TTP}

At increased shear stress, vWF becomes susceptible to proteolysis by ADAMTS13, which cleaves vWF within the A2 domain (between Y1605 and M1606), decreasing vWF multimer size and GPIb-dependent platelet adhesion (13). Shear stress applied to large multimers anchored to endothelial cells induces conformational changes to A2, facilitating proteolysis not only by ADAMTS13, but also by granzyme B from cytotoxic lymphocytes and other proteases that cleave in the same region (14-16). Further, the cleavage point in large vWF multimers is predominantly in the central region of the multimer rather than near the ends (13), maximizing the reduction of vWF multimer size and hence their prothrombotic tendency. This means the downsizing of multimers by ADAMTS13 and the regulation of ADAMTS13 activity are carefully balanced to optimize vWF multimer size within a healthy range for normal function. Indeed, dysfunctional ADAMTS13, due to autoimmune or other causes, leads to thrombotic problems. TTP is the bestknown clinical example of ADAMTS13 dysfunction; it is a multifactorial disease where defective ADAMTS13 activity due to autoantibodies, mutations, or deficiency can result in ULVWF multimers, thrombocytopenia due to vWF-binding platelets, and multiple thrombotic microangiopathies $(1,13)$. Acute thrombotic episodes can be triggered by other prothrombotic conditions, such as increased inflammation $(17,18)$.

\section{Breaking the bonds}

Whereas ADAMTS13 reduces vWF multimer size by proteolysis within A2, essentially the same effect can be achieved by chemical reduction. In this regard, ADAMTS13 has recently been shown to reduce $\mathrm{vWF}$ multimer size in a second way, in that it can also facilitate disulfide bond reduction of vWF (19). Reducing intermolecular disulfide bonds leads to shorter multimers, but there has been little evidence regarding whether this process could either be relevant in vivo or utilized as a therapeutic approach. Chen and colleagues (2) examined the effects on the size and reactivity of ULVWF of a free

\section{Figure 1}

Regulation of vWF-dependent thrombosis under shear conditions. vWF is normally expressed as ultralarge, prothrombotic multimers on activated endothelial cells. These ultralarge multimers are prothrombotic in that they support the initial adhesion of circulating platelets via an interaction with the GPIb $\alpha$ subunit of the platelet receptor GPIb-IX-V, leading to microthrombi formation. In healthy individuals, ADAMTS13 cleaves within the vWF-A2 domain, releasing smaller vWF multimers into the circulation that no longer spontaneously bind platelets. In patients with TTP, ADAMTS13 activity is deficient, resulting in vascular microthrombi. In their study in this issue of the $\mathrm{JCl}$, Chen et al. (2) show that the drug NAC can also decrease vWF multimer size by reducing intersubunit disulfide bonds to form smaller vWF multimers, following initial reduction of the intrasubunit disulfide (C1272-C1458) of the A1 domain.

thiol-containing reducing agent, NAC, which is already used clinically, but not for the treatment of TTP. NAC was shown to reduce the intra-A1 disulfide bond as well as intersubunit disulfide bonds in vWF, altering both the mean multimer size and platelet adhesive function, as shown in a number of functional assays, for example, shear-dependent occlusive aggregate formation in the PFA100 system. The influence of shear forces on NAC-induced demultimerization of vWF was demonstrated both in vitro and in vivo (in mice) and occurred efficiently upon exposure to shear. NAC treatment also decreased the appearance of platelet-ULVWF strings on the surface of activated human endothelial cells. In other experiments, NAC was injected into ADAMTS13-deficient mice, which have abnormally large vWF multimers. NAC, at doses comparable to those achieved in plasma of patients treated for acetaminophen overdose, was shown to profoundly inhibit the formation of ionophore-provoked mesenteric thrombi seen in mice without NAC treatment (2). Importantly, there was no measurable impact on tail-bleeding time in NAC-treated mice (2). This is consistent with the fact that clinical use of NAC in patients with either chronic obstructive lung disease or acetaminophen toxicity is not known to be associated with bleeding and implies that NAC could be used to treat pathological shear-related thrombosis in the context of TTP. 


\section{Outstanding issues}

There are, however, a number of caveats to the view that NAC could be used to treat conditions such as TTP. As shown by Chen et al. and others, NAC has an inhibitory effect on platelet function beyond that involving GPIb/vWF interaction, such as inhibition of adenosine diphosphate- and collageninduced platelet aggregation and nitric oxide scavenging by reactive oxygen species $(2,20)$. NAC also impedes coagulation (21). The lack of effect of NAC on bleeding time therefore needs to be treated with caution, given that acute episodes of TTP are associated with thrombocytopenia and that Chen and colleagues did not assess NAC efficacy in an acute animal model of TTP. Nevertheless, their work (2) suggests a potential new therapeutic approach that may show superiority and a better safety profile than current clinical treatment. The results of future preclinical evaluation of NAC in animal models of TTP are therefore eagerly awaited.

\section{Acknowledgments}

The authors gratefully acknowledge research support from Science Foundation Ireland and the National Health and Medical Research Council of Australia.

Address correspondence to: Michael C. Berndt, Biomedical Diagnostics Institute, Dublin City University, and Royal College of Surgeons in Ireland, Dublin, Ireland. Phone: 353.1.7007658; Fax: 353.1.7006558; E-mail: michael.berndt@dcu.ie.

1. George JN. How I treat patients with thrombotic thrombocytopenic purpura: 2010. Blood. 2010; 116(20):4060-4069.

2. Chen J, et al. N-acetylcysteine reduces the size and activity of von Willebrand factor in human plasma and mice. J Clin Invest. 2011;121(2):593-603.

3. Lenting PJ, Pegon JN, Groot E, de Groot PG. Regulation of von Willebrand factor-platelet interactions. Thromb Haemostas. 2010;104(3):449-455.

4. Sadler JE. von Willebrand factor assembly and secretion. J Thromb Haemostas. 2010;7 suppl 1:24-27.

5 . Yago $T$, et al. Platelet glycoprotein Ib $\alpha$ forms catch bonds with WT VWF but not with type 2B von Willebrand Disease VWF. J Clin Invest. 2008; 118(9):3195-3207.

6. Kim J, Zhang CZ, Zhang X, Springer TA. A mechanically stabilized receptor-ligand flexbond important in the vasculature. Nature. 2010; 466(7309):992-995

7. Fujimura Y, et al. von Willebrand factor. A reduced and alkylated $52 / 48-\mathrm{kDa}$ fragment beginning at amino acid residue 449 contains the domain interacting with platelet glycoprotein Ib.J Biol Chem. 1986;261(1):381-385

8. Andrews RK, Gorman JJ, Booth WJ, Corino GL, Castaldi PA, Berndt MC. Cross-linking of a monomeric 39/34-kDa dispase fragment of von Willebrand factor (Leu-480/Val-481-Gly-718) to the N-terminal region of the $\alpha$-chain of membrane glycoprotein Ib on intact platelets with $\mathrm{b}$ is(sulfosuccinimidyl)suberate. Biochemistry. 1989; 28(21):8326-8336

9. Huizinga EG, et al. Structures of glycoprotein Ib $\alpha$ and its complex with von Willebrand factor A1 domain. Science. 2002;297(5584):1176-1179.

10. Dumas JJ, et al. Crystal structure of the wild-type von Willebrand factor A1-glycoprotein Ib $\alpha$ com- plex reveals conformation differences with a complex bearing von Willebrand disease mutations. J Biol Chem. 2004;279(22):23327-23334.

11. Shen Y, et al. Leucine-rich repeats 2-4 (Leu60Glu128) of platelet glycoprotein $\mathrm{Ib} \alpha$ regulate shear-dependent cell adhesion to von Willebrand factor. J Biol Chem. 2006;281(36):26419-26423.

12. Dong JF, et al. ADAMTS-13 rapidly cleaves newly secreted ultralarge von Willebrand factor multimers on the endothelial surface under flowing conditions. Blood. 2002;100(12):4033-4039.

13. Zhou Z, Nguyen TC, Guchhait P, Dong JF. Von Willebrand factor, ADAMTS13, and thrombotic thrombocytopenic purpura. Semin Thromb Hemost. 2010;36(1):71-81.

14. Buzza MS, et al. Antihemostatic activity of human granzyme B mediated by cleavage of von Willebrand factor. J Biol Chem. 2008;283(33):22498-22504.

15. Raife TJ, et al. Leukocyte proteases cleave von Willebrand factor at or near the ADAMTS13 cleavage site. Blood. 2009;114(8):1666-1674.

16. BaldaufC, et al. Shear-induced unfolding activates von Willebrand factor A2 domain for proteolysis. J Thromb Haemost. 2009;7(12):2096-2105.

17. George JN. ADAMTS13 alone. Blood. 2010; 116(12):2003-2004.

18. Feys HB, et al. Thrombotic thrombocytopenic purpura directly linked with ADAMTS13 inhibition in the baboon (Papio ursinus). Blood. 2010; 116(12):2005-2010.

19. Yeh HC, et al. Disulfide bond reduction of von Willebrand factor by ADAMTS-13. J Thromb Haemost. 2010;8(12):2778-2788.

20. Anfossi G, Russo I, Massucco P, Mattiello L, Cavalot F, Trovati M. N-acetyl-L-cysteine exerts direct antiaggregating effect on human platelets. Eur J Clin Invest. 2001;31(5):452-461.

21. Jepsen $\mathrm{S}$, Hansen $\mathrm{AB}$. The influence of $\mathrm{N}$-acetylcysteine on the measurement of prothrombin time and activated partial thromboplastin time in healthy subjects. Scand J Clin Lab Invest. 1994; 54(7):543-547. 